\title{
Pour une histoire du désir
}

\author{
Yves FERROUL
}

Sexologue, Agrégé de Lettres, Docteur en Lettres

\section{RESUME}

Ce quî frappe dans une exploration de l'histoire du désir sexuel, c'est le contraste entre l'omniprésence de ce sentiment dans les sociétés humaines, quelle que soit l'époque considérée, et la représentation négative que certains, philosophes et religieux, en ont constamment et systématiquement donnée.

Le désir a ainsi été disqualifié d'office par ceux à qui répugnait la part animale de l'homme : les bêtes n'agissent que pour satisfaire leurs besoins physiques ; I'homme moral doit donc s'en distinguer en refusant d'agir pour le plaisir. Telle est la conviction des stoïciens au premier siècle de notre ère, vite rejoints par les théologiens de la jeune religion chrétienne. Pour Cicéron ou Sénèque, " rechercher un acte à cause du plaisir qui lui est lié est un abus ". Ils appliquent ce principe aussi bien à l'alimentation qu'aux soins du corps : les animaux sans raison vivent pour manger, alors que l'être humain moral mange pour vivre, lui ; la nourriture et l'entretien du corps ont pour but la santé et non pas le plaisir... Dans le même esprit, la sexualité ne se justifie que si elle a pour finalité la reproduction.

Pourtant, constate déjà saint Augustin, ce n'est pas ainsi que les humains se motivent à agir, et ce Père de l'Église doit reconnaitre qu'il n'a jamais rencontré de mari capable d'affirmer "n'avoir eu de rapports que dans l'espoir d'engendrer" (Sur le bien conjugal).

D'autres penseurs ont été plus nuancés, et distinguent d'une part un désir acceptable, restant sous la domination de la raison et poussant à l'action positive, et d'autre part un désir dégradant, irrationnel, aboutissant à la passion (toujours imaginée déraisonnable). Seul le second est à fuir ; le premier est source de bonheur et d'épanouissement, il reste le moteur habituel de l'action humaine.

Les penseurs chrétiens du Moyen Âge, attentifs au vécu des croyants, finiront par admettre timidement que la mémoire, comme caractéristique humaine, est un des plus beaux dons du Créateur, et qu'elle permet aux humains de se souvenir des plaisirs passés, donc d'a- voir légitimement le désir de les renouveler : le coït sans projet de procréation apparaît alors comme conforme au plan divin. "Si quelqu'un mange une poire ou un beau fruit pour le seul plaisir, il ne pèche pas. Il en est de même quand il connaît sa femme pour le seul plaisir" affirmera même un théologien, à contre courant de l'opinion religieuse dominante.

Mais, loin des discussions théoriques, les individus témoignent de leur profond attachement au jeu avec le désir, que ce soit dans la société mouvante de la Renaissance, à la cour du roi Soleil, dans les salons des Lumières ou encore, contredisant nos idées reçues, dans le nouveau monde industriel du XIXe siècle.

Aujourd'hui, alors que les responsables religieux ont toujours massivement une conception stoïcienne du plaisir et du désir, la pensée moderne a évolué dans sa compréhension de la place de ce dernier dans l'expérience humaine. Elle lui reconnaît un rôle moteur, source primordiale de l'épanouissement et stimulus puissant de toute créativité.

L'histoire du désir sexuel s'offre alors comme la recherche des étapes de l'enrichissement que l'espèce humaine a apporté au simple instinct animal, et celle des formes prises par les réactions individuelles ou sociales à sa mise en œuvre.

Mots clés : désir, sexualité humaine, plaisir, morale

\section{Correspondance :}

Dr Yves FERROUL - 339 rue de la Frête, 59830 Louvil Tel 03.20.79.51.10 - Email yves.ferroul@wanadoo.fr 
L'histoire du désir est caractérisée par deux faits constants: l'universalité de la présence de ce sentiment sur lequel nous avons des témoignages dès les premiers textes de notre civilisation occidentale ; et l'ambiguïté des attitudes qu'il suscite à toutes les étapes de l'évolution des sociétés humaines, partagées de façon permanente entre acceptation positive et même ludique d'une part, rejet dépréciatif et culpabilisant d'autre part.

Aux origines, la civilisation babylonienne nous a légué de nombreuses tablettes où se lit le désir sexuel de la personne amoureuse. Une trentaine d'entre elles sont des prières de femmes demandant à la déesse que leur amant tienne bon jusqu'au bout afin de leur assurer tout le plaisir physique qu'elles étaient en droit d'attendre de lui. Les jeux avec le désir sont évoqués, pour lesquels on préconise, au lieu de la chambre à coucher, le toit-terrasse de la maison, le seuil de la porte, les champs ou les vergers, les lieux déserts ou les chemins sans issue, ou encore les tavernes. Les positions aussi peuvent se varier, les ouvrages évoquant l'amour debout, sur une chaise, en travers du lit, en prenant la partenaire par derrière, en la sodomisant, en étant chevauché par elle [5]. II est étonnant de constater comment ces conseils et ces pratiques d'il y a 3000 ans sont toujours d'actualité. Les désirs et les fantasmes de ces lointains ancêtres nous semblent beaucoup plus proches que les autres caractéristiques de leurs vies.

\section{L'ANTIQUITÉ CLASSIQUE}

Dans le monde grec, la pensée philosophique ne tient pas en grande estime le désir. Au mieux. Platon va en distinguer une forme acceptable, quand le désir reste sous la domination de la raison et qu'il pousse à l'action positive, et une forme dégradante, irrationnelle, aboutissant à la passion qui est toujours déraisonnable.

Mais, le plus généralement, les philosophes narrivent pas à accorder au désir une composante positive, et ils préfèrent appeler simplement "appétit" cette envie qui reste soumise à la raison et qui est l'apanage du sage.

Cependant les écrivains donnent constamment des exemples positifs de désir sexuel, comme Aristophane (445-386 av. J.-C.) dans sa pièce Lysistrata où il met en scène des femmes cherchant un moyen de faire pression sur leurs maris afin d'arrêter la guerre : l'une d'elles propose la grève du sexe. "Eh bien ? Vous me tournez le dos ? Pourquoi ? Hé, vous autres, vous faites la moue, vous secouez la tête, pourquoi ? - Autre chose, tout ce que tu voudras d'autre! Je veux bien, s'il faut, passer à travers le feu ! Oui, j'aime mieux ça que ton truc ! Le sexe, Lisette chérie, il n'y a rien qui vaille ça ! - Moi aussi, j'aime mieux passer par le feu. Ah ! Le beau sexe que le nôtre! II ne pense qu'à se faire toujours boucher le petit coin ! - Mais il y a de quoi se languir, pour une femme, à dormir toute seule! - Mais si on restait tapies chez nous, le museau fardé, toutes nues dans nos chemisettes, à rôder autour de nos hommes, il monterait dru le désir de nous culbuter ! Et au lieu de nous laisser approcher, on se déroberait. Ils auraient vite fait de conclure la paix, crois-moi ! - Mais dis-moi, ma jolie, et s'ils nous laissent tomber, nos hommes ? - Alors, comme disait l'autre, faute d'outils, on se travaillera à la main ! - Et s'ils nous empoignent pour nous traîner de force dans la chambre ? - Cramponne-toi aux portes ! - Et s'ils nous rossent ? - Il faut leur laisser faire leur affaire, en rechignant. Ils n'ont pas de plaisir à la chose, si on n'est pas consentantes. Jamais un homme n'aura du bon temps si la femme n'y met pas du sien !" [2].

Les jeux de séduction ne sont pas les seuls, et Hérondas, un auteur du Ille siècle avant J.-C., met en scène des femmes qui se confient entre amies les bonnes adresses: "Je t'en supplie, ma chère, dis-moi qui t'a façonné cet olisbos écarlate ? - J'ai déniché un petit cordonnier ... II travaille en chambre, pour vendre en cachette. Mais son travail, quel travail ! Pour moi, d'envie, à le voir, les yeux me sortaient de la tête. Les hommes n'atteignent pas cette rigidité. Et il n'y a pas seulement cela, mais la douceur, un rêve ..." [14].

Ces deux exemples suffisent pour montrer que, dans la société grecque antique, le désir est là, vivant et très ludique.

Dans le monde romain, la très grande facilité de la vie sexuelle, avec l'omniprésence des esclaves dont le corps est la propriété des maîtres, en fait une activité banale dont il est de bon ton de se montrer détaché : Cicéron (106-43 av. J.-C.) veut consacrer toute son énergie à la philosophie, et son secrétaire n'est là que pour l'hygiène, le soulagement d'une nécessité physique. Comme le confirme Sénèque, le devoir d'un esclave est de se mettre à quatre pattes quand son maître le désire ... Pour les intellectuels de cette époque, on assouvit un désir, on ne joue pas avec le désir.

Mais tout le monde ne partage pas cette philosophie. Concrètement, les femmes romaines, elles, castraient leurs plus beaux esclaves mâles adultes afin de pouvoir en jouir sans risque et combler ainsi leur désir $[15,17]$.

Les empereurs, de leur côté, multiplient les inventions érotiques dont les historiens nous cachent peu de choses : il est vrai que leur toute puissance leur offrait des possibilités illimitées [27].

C'est dans ce contexte qu'il faut interpréter l'œuvre d'Ovide (43 av. J.-C. - 18 apr.), L'Art d'aimer : comment échapper à la routine de la vie sexuelle, à la satiété, quand on a la possibilité de jouir sur-le-champ avec qui l'on veut, comme on veut, et à chaque fois que l'on on en a envie?

Les Romains, certains romains, avaient un réel problème de désir, et le poète imagine de proposer de recourir à la drague, à la séduction : préparer des plans de rencontre, des tactiques de courtisement, ne pas se contenter de jouir mais être attentif à procurer la jouissance de l'autre, autant de projets absurdes, comiques, pour une classe à qui pratiquement personne ne peut dire non, mais qui pouvaient peut-être enfin pimenter un désir par trop banalisé [19]. 


\section{PREMIERS BOULEVERSEMENTS}

Au début de notre ère, des changements profonds vont affecter la compréhension du désir.

Ce sont d'abord les stoïciens qui lui donneront une place différente dans la morale individuelle. Leur postulat est que "il ne faut rien faire pour le plaisir", puisqu'alors on ressemblerait aux animaux qui n'agissent que pour satisfaire leurs besoins physiques. À partir de ce principe général, le philosophe Sénèque (2 av. J.-C. - 65 apr.) définit, parmi les comportements humains, ceux qui recherchent le plaisir, donc ceux qui sont pour lui "contre nature" : nager dans une piscine chauffée, cultiver des plantes dans une maison ou sous abri, s'efforcer de paraître jeune, boire l'estomac vide, se raser ... Le jeu avec le désir et le plaisir qui consiste à transformer les conditions qu'offre directement la Nature reçoit une qualification péjorative, dans un contexte moral. Or, on voit bien que ces comportements sont tout simplement "artificiels" : et le simple fait de se contenter de dire qu'ils sont "artificiels" au lieu de "contre nature" leur ôterait tout caractère immoral car l'espèce humaine a spontanément multiplié les artifices dans tous les domaines. En fait, refuser toute élaboration du désir revient à ramener l'être humain au comportement animal élémentaire : un animal se baigne dans l'eau telle qu'elle est, seul un être humain peut imaginer de la chauffer, et seul il le fait.

Dans la logique de son raisonnement, Sénèque propose aussi de prendre la maîtrise du désir sexuel comme critère de moralité : puisqu'elle est, de toutes les maîtrises de soi, la plus difficile à mettre en oeuvre, sa qualité serait bon signe de la qualité morale d'une personne. Dans le couple, écrit-il, "il est honteux d'aimer abusivement son épouse. Le sage laisse la raison et non le sentiment guider l'amour qu'il porte à son épouse. II résiste à l'assaut des passions et ne se laisse pas entraîner sans contrôle à l'acte conjugal. Rien n'est plus corrompu que d'aimer son épouse comme une femme adultère ... II faut se conduire en époux avec sa femme et non en amant" [26]. "L'idée que le rapport conjugal se doive d'être un acte de procréation, faute de quoj il tombe dans la catégorie négative du plaisir, la catégorie d'amour n'entrant pas en ligne de compte, a durablement influencé le christianisme" [21:19].

À la même époque, Jésus prêchait que "Quiconque regarde une femme pour la désirer a déjà commis, dans son cœur, l'adultère avec elle" : il considère donc comme faute morale le désir intérieur, même en l'absence de tout acte effectif (Évangile selon saint Matthieu, 5, 27-28). Pourtant, constatera déjà saint Augustin (354-430), ce n'est pas en ignorant le désir que les humains se motivent à agir sexuellement, et ce Père de l'Église doit reconnaître qu'il n'a jamais rencontré de mari capable d'affirmer "n'avoir eu de rapports que dans l'espoir d'engendrer" [3].

Cependant, avec Jésus, la boucle est bouclée, et le désir allait être disqualifié moralement pour des siècles même si, bien sûr, cette disqualification ne l'a pas fait disparaître de la vie quotidienne des humains.

\section{LES TROUBADOURS ET LA JOIE DE DÉSIRER}

Il faut attendre le Xlle siècle et la poésie les troubadours pour voir proclamée et diffusée une autre conception du désir.

Dans un contexte de facilités sexuelies fort semblable à celui qu'avait connu Ovide, et d'ailleurs après avoir médité la leçon du poète romain, les auteurs de chansons vont dénigrer un passage à l'acte trop aisé et peu flatteur, pour valoriser un jeu d'exacerbation du désir, de la frustration, seul capable de porter à l'incandescence les âmes les mieux trempées. Faire la cour, se comporter en chevalier servant, traiter la femme en dame de son cœur, toutes ces attitudes nouvelles vont marquer durablement la société occidentale.

Avec cette promotion du désir, Héloïse, mère abbesse séparée de son mari depuis des années, après leur commune entrée au couvent, pourra alors écrire à son époux, Abélard : "les voluptés chères aux amants que nous avons goûtées ensemble me furent douces et je ne peux ni les détester, ni les chasser de ma mémoire. Où que je me tourne, elles s'imposent à mes yeux avec les désirs qui les accompagnent. Même quand je dors, elles ne m'épargnent pas leurs illusions. En pleine solennité de la messe, lorsque la prière doit être plus pure, les représentations obscènes de ces voluptés captivent totalement mon âme si bien que je m'abandonne plus à ces turpitudes qu'à la prière. Alors que je devrais gémir des fautes commises, je soupire plutôt après les plaisirs perdus. Non seulement les actes réalisés, mais aussi les lieux et les moments où je les ai vécus avec toi sont à ce point fixés dans mon esprit que je refais tout avec toi dans les mêmes circonstances, et même dans mon sommeil ils ne me laissent pas en paix. Souvent les pensées de mon cœur peuvent être comprises aux mouvements de mon corps, des mots m'échappent malgré moi." [13].

\section{OMNIPRÉSENCE DU DÉSIR}

Pendant tous ces siècles, le désir sexuel est là, bien présent dans la société et reconnu comme tel. Les médecins en soignent les troubles [12:45-61], conseillant de consacrer du temps aux préliminaires et d'éviter toute hâte, puis d'"écouter des contes, des chants et d'autres choses semblables qui amènent à l'amour, qu'ils regardent de très belles dames, qu'ils imaginent longuement l'acte sexuel jusqu'à ce qu'ils se sentent réconfortés" [24]. Ou encore : "Que celui qui souffre d'une diminution du coït s'habitue à ne rougir ni de l'acte sexuel, ni de l'audition de récits à son sujet. Que soient lus devant lui des livres qui traitent de l'amour charnel, des actes vénériens et de leurs figures, afin qu'il ne rougisse ni d'en parler ouvertement, ni d'en entendre parler, ni d'accomplir le coït quand il le désire. Qu'il imagine souvent les plaisirs vénériens et qu'il pense qu'engendrer son semblable constitue pour les êtres vivants une œuvre très naturelle" [9].

Les moralistes veulent canaliser le désir sexuel et remarquent que "vers 14 ans, la jeune fille commence à désirer 
l'acte sexuel, mais, dans ce désir, elle n'émet pas ; plus elle se livre à des frottements manuels, plus elle éprouve d'appétit, de sorte que par une telle pratique l'humeur spermatique est attirée ... C'est pourquoi certaines jeunes filles âgées d'environ 14 ans ne peuvent être satisfaites : si elles ne sont pas pourvues d'un homme, elles imaginent l'acte sexuel et le pénis viril, et en arrivent à se frotter avec les doigts ou avec d'autres instruments jusqu'à ce que I'humeur spermatique sorte ainsi que la chaleur ; de cette façon elles tempèrent leurs parties génitales et deviennent plus chastes" [1]. Certains penseurs chrétiens du Moyen Âge, attentifs au vécu des croyants, finiront même par admettre timidement que la mémoire, en tant que caractéristique humaine, est un des plus beaux dons du Créateur. Comme elle permet aux humains de se souvenir des plaisirs passés, elle suscite légitimement le désir de les renouveler : le coït sans projet de procréation apparaît alors conforme au plan divin : "si quelqu'un mange une poire ou un beau fruit pour le seul plaisir, il ne pèche pas. II en est de même quand il connaît sa femme pour le seul plaisir" affirmera ainsi un théologien, à contre courant de l'opinion religieuse dominante.

Pour ces penseurs, le désir est donc pleinement naturel, même le désir homosexuel. II ne viendrait à l'esprit d'aucun responsable de dire qu'un tel désir est le signe d'un esprit tordu. Par exemple, si les dirigeants de monastères mettent leurs moines en garde, c'est parce qu'ils pensent que le désir sexuel est naturel, mais que les voeux religieux engagent dans une autre voie : "Il arrive souvent chez les jeunes gens que, même s'ils exercent une rigoureuse ascèse, l'éclat brillant de leur âge éveille par son épanouissement le désir de ceux qui les entourent. Si donc il est parmi vous un jeune homme doué de beauté physique, qu'il dissimule ses attraits ... Ne vous asseyez pas près de lui ; pendant le sommeil, que vos vêtements ne touchent pas les siens ... Quand il vous parle ou qu'il chante en face de vous, dirigez le regard vers le sol ... Ne vous trouvez jamais seul avec lui ... " [4].

À la Renaissance, Brantôme rédigera une enquête très documentée sur ce qui peut susciter le désir féminin, sur les formes qu'il peut prendre, sur les façons variées que l'imaginaire infini des femmes trouve pour le satisfaire, sur les réactions défensives qu'il provoque chez les hommes, souvent désemparés. En témoigne, entre mille exemples, cet hymne à la puissance du désir féminin : "si être au-dessus me plaît, je m'installe et reste ainsi par plaisir. Mais quand je n'en ai pas envie, je me fais servir par vous comme d'un esclave ou d'un galérien, et je vous fais tirer au collier comme un vrai cheval de labour, et vous, travaillant, peinant, suant, haletant, vous vous efforcez de faire les corvées et les efforts que je veux tirer de vous. Cependant, moi, je suis couchée à mon aise, je ris et je tire mon plaisir à vous voir vous dépenser. Et, après en avoir très bien passé ma fantaisie, je laisse là mon galant, recru, affaibli, sans ressort, qui n'en peut plus, et n'a besoin que d'un bon repos et d'un bon repas, pour refaire ses forces. Tandis que moi, je ne m'en ressens pas, et n'ai d'autre mal sinon de souhaiter quelque autre galant qui m'en donne autant, avec comme peine de le faire rendre comme le premier. Et, ainsi, ne me rendant jamais, mais faisant rendre mon doux ennemi, je remporte la vraie victoire et la vraie gloire" [6 : Premier discours].

À l'époque classique, le duc de Saint-Simon actualisera avec des exemples de la cour de Louis XIV la documentation de son prédécesseur, et insistera sur le fait que la liberté du désir est plus une question de rang social que de domination masculine : les femmes de rang supérieur ou ayant des appuis puissants sont maîtresses de leurs désirs et usent sans vergogne de leur pouvoir. On peut aussi retenir de ces anecdotes les cas qui troublent le plus notre sensibilité. Telle la mésaventure survenue à une jeune mariée et que celle-ci racontera bien plus tard, afin de faire rire ses auditeurs, alors que nos modernes y verraient la source d'un traumatisme profond du désir : "Françoise de Noailles avait épousé un duc de soixante quatre ans et apoplectique, et elle ravissait ses auditeurs en leur contant 'comment elle avait la honte d'avoir eu ses enfants moyennant des valets de chambre qui remuaient et soutenaient' son mari" [23].

Le XVIIlème siècle, ramenant avec la Régence la facilité sexuelle, crée les libertins qui exploreront, comme Casanova [7], la variété des jeux du désir, ce dont témoigne le célèbre roman Les Liaisons dangereuses [16]. Tandis que Sade théorisera la recherche d'une voie différente, fondée sur l'excès, la violence, la souffrance, et que les auteurs n'avaient évoquée qu'incidemment jusqu'alors, malgré l'ancienneté des témoignages (de Néron à Gilles de Rais).

\section{LE CAS PARTICULIER DU XIXe SIÈCLE}

Le XIXe siècle va se distinguer par deux traits. Le premier est l'extension du domaine du désir sexuel : alors que la réflexion sur la sexualité ne concernait jusqu'alors pratiquement que les adultes et particulièrement les personnes mariées, ce siècle va se focaliser sur le désir des enfants et des adolescents (répression de la masturbation) et sur celui des marginaux (délinquants, malades psychiatriques, prostituées) rendant la sexualité omniprésente et envahissante. On voit du désir sexuel partout, ce qui sera finalement théorisé par Freud avec l'invention de la libido : cette substance chimique unique, sorte d'hormone libidinale sécrétée dans les différentes parties du corps, déterminerait une pulsion sexuelle fondamentalement une et identique à elle-même sous des habillages variés qui tiennent à la partie du corps concernée. Par exemple, apparaissant au niveau bucco-lingual chez le nourrisson, cette substance sexuelle sous-tendrait la sexualité orale, et légitimerait une telle appellation (au lieu de celle de plaisir oral tout simplement) $[18,11]$.

Le second trait est la volonté de renverser la conviction profondément ancrée depuis toujours que les femmes ont un désir bien plus fort que les hommes : une minorité très active, relayée efficacement par les responsables religieux, affirmera que la femme idéale n'a pas de désir et que c'est l'homme qui "ne pense qu'à ça". Malgré une résistance largement majoritaire de médecins et de "féministes" récla- 


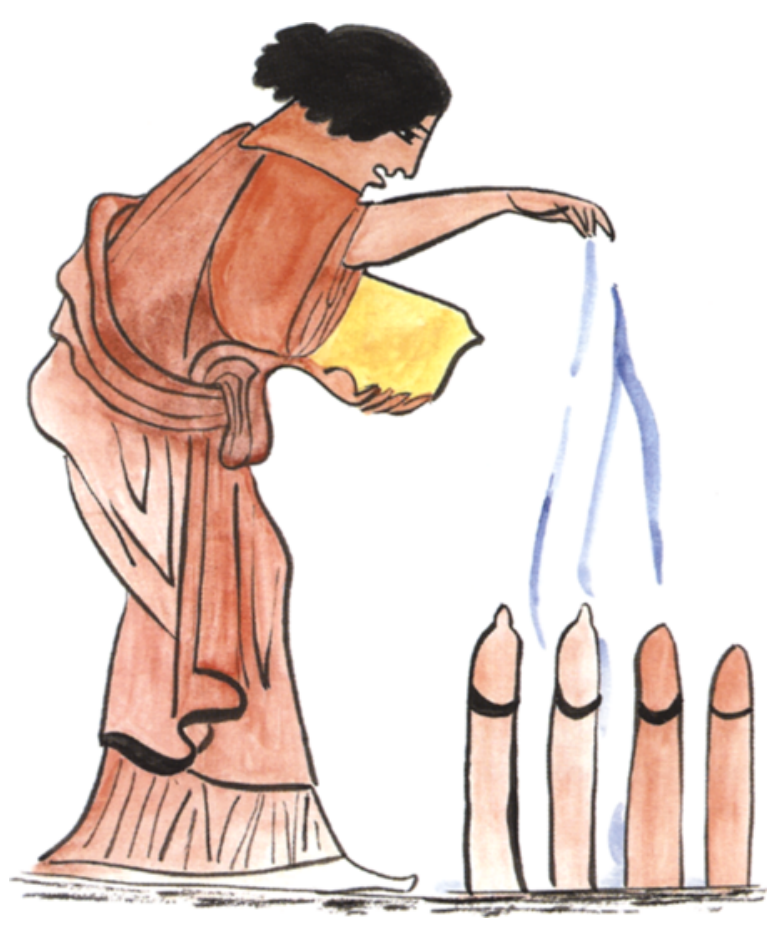

Figure 1 : Dessin d'après un vase de 430 environ avant J.C. (Dessin d'Yves Ferroul).

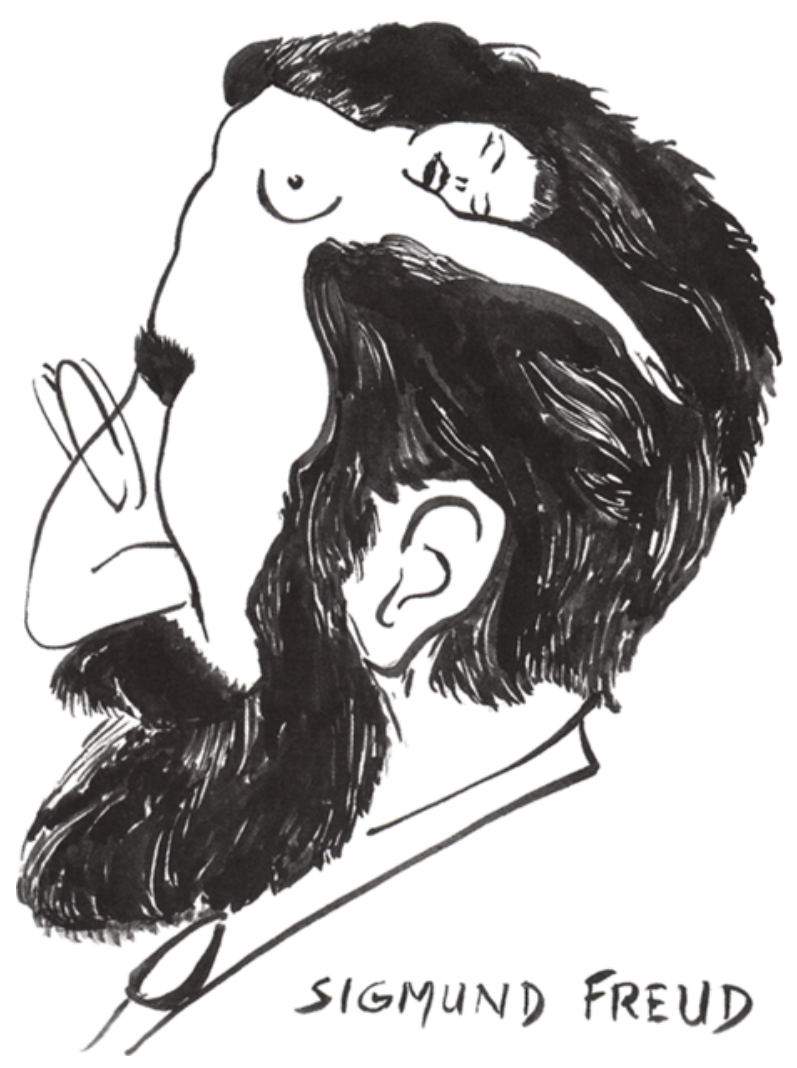

Figure 3 : Freud (Dessin d'Yves Ferroul).

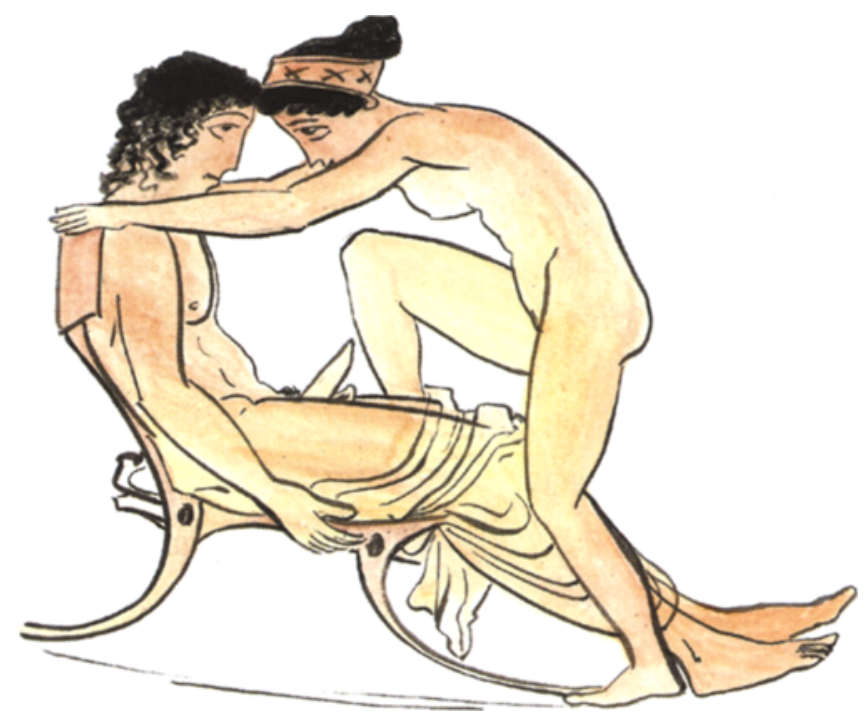

Figure 2 : Dessin d'après une cruche du Véme siècle avant J.C. (Dessin d'Yves Ferroul).

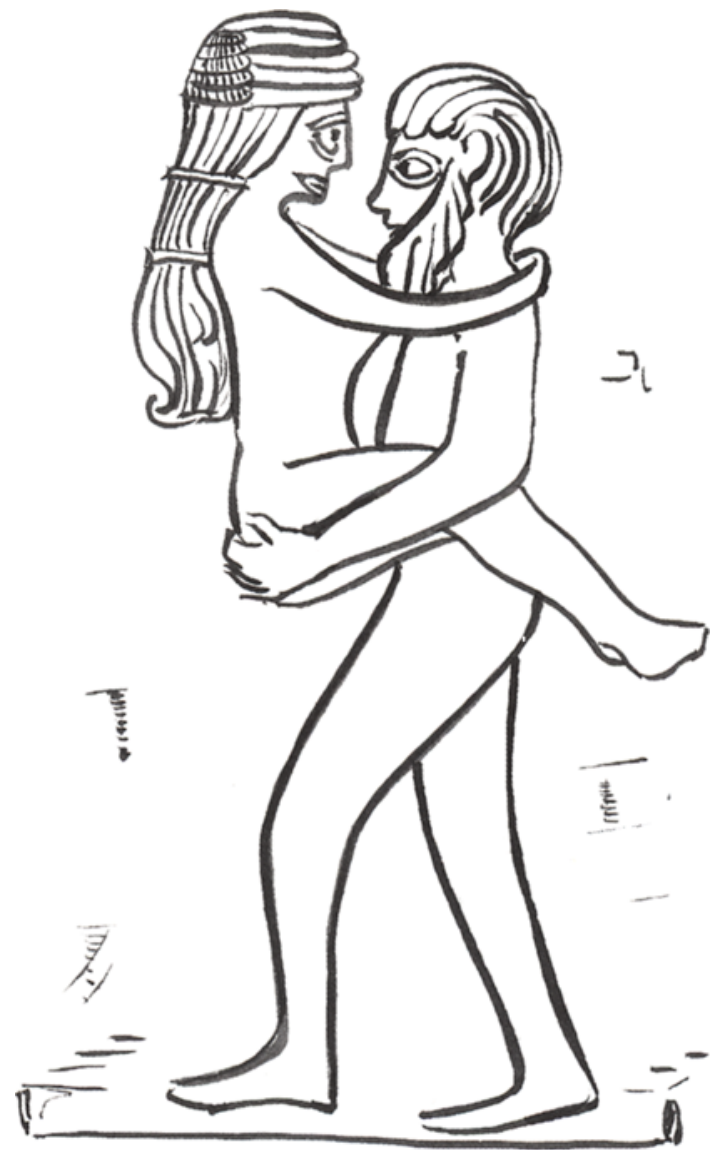

Figure 4 : Rite de Fertilité (Dessin d'Yves Ferroul). 
mant le droit à l'orgasme pour la femme mariée [28], certains défendront l'idée que "le modèle le plus parfait de la femme d'intérieur, de la mère, est celle qui est pleine d'attentions délicates, prête à tout sacrifice raisonnable et tellement pure de cœur que tout désir sexuel lui est inconnu ; qui éprouve plutôt une répulsion à cet égard, mais qui est si dévouée à son mari bien-aimé qu'elle est toute disposée à lui sacrifier ses sentiments et ses désirs" [22].

\section{LE DÉSIR ET LES RESPONSABLES DE L'ÉGLISE CATHOLIQUE}

Au milieu du XIXe siècle, un ouvrage de référence pour les confesseurs évoque à plusieurs rubriques le problème du désir. Les interprétations sont toujours en accord avec la pensée stoïcienne : "une jeune fille de seize ans qui mange souvent des fruits du jardin de la maison de son père, ou des confitures que l'on fait chez elle, seulement pour contenter son goût ... pèche par intempérance ... puisqu'elle ne mange ces fruits ou ces confitures que par le désir qu'elle a de satisfaire son goût et de se procurer la délectation sensuelle qu'elle y trouve et qu'elle se propose comme fin, sans qu'elle en ait aucun besoin" [20 : article "Intempérance"]. De même "les baisers excitants et les attouchements intimes entre époux ne sont licites que comme préliminaires au rapport complet. Ils sont des péchés dans les autres cas, morteis s'ils aboutissent à des orgasmes sans pénétration" [20 : "Attouchement et baiser"].

Cent cinquante ans plus tard, rien n'est changé. Le Catéchisme de l'Église catholique [8], en 1992, raisonne toujours selon les mêmes principes. "Le désir sexuel est moralement désordonné quand il est recherché pour luimême" (paragraphe 2351) : comme pour Sénèque ou Cicéron, le plaisir pour le plaisir est immoral. S'il est bien dit que "la sexualité est source de joie et de plaisir ... Les époux ne font rien de mal en recherchant ce plaisir et en en jouissant" (2362), la formule ne signifie pas la liberté de la recherche car la sexualité reste soumise à sa finalité de procréation : sinon elle est désir désordonné, c'est-à-dire luxure (2351). Les actes doivent rester "honnêtes et dignes" (une femme qui gémit perd-elle sa dignité ? la levrette est-elle une position digne ? quel est le sens d'une fellation digne ?), doivent être maintenus "dans les limites d'une juste modération" (une fois par jour, par semaine, par mois ? D'une durée de dix minutes, d'une heure ? Est-ce que faire l'amour trois heures de suite c'est respecter une juste modération, donc respecter la morale ?).

De plus il n'existe qu'une seule alternative : "ou l'homme commande à ses passions, ou il se laisse asservir par elles" (2339). Si "commander à ses passions" signifiait agir en être humain responsable, l'alternative serait acceptable. Or, pour le catéchisme, l'homme commande à ses passions en "se délivrant de toute servitude", par une ascèse qui l'amène à imiter la pureté du Christ (2345), en exerçant une chasteté qui s'épanouit dans l'amitié (2347) ! L'idéal de la sexualité serait ainsi l'amour du prochain : c'est-à-dire que le désir et la sexualité sont évacués du projet de vie sexuelle! Des théologiens chrétiens s'insurgeront contre cette façon de parler du désir humain : "ils se refusent à voir que les vagues de la passion n'engloutissent pas la barque du moi humain, mais la portent" écrit Eugène Drewermann, qui défend la valeur positive du désir [10]. Et une femme, théologienne catholique mariée, Uta Ranke, intitule "comment tyranniser les gens mariés par l'interdiction du plaisir" la façon dont les responsables religieux traitent le désir, et parle de "désastre de la morale catholique", de "volonté de plier les époux à un démontage du plaisir" : de telles absurdités sont pour elle le fait d'hommes totalement incompétents dans la matière qu'ils traitent [21].

Le fossé s'est creusé entre les prescriptions élaborées au moyen de raisonnements théoriques coupés de l'expérience vivante, auxquelles s'accrochent certains moralistes et théologiens, d'une part, et, d'autre part, la population dans son ensemble qui, grâce à la "société de consommation", est entrée dans une civilisation où le plaisir est devenu un objectif accessible dans ce monde, libérant et cautionnant le désir : la publicité notamment, caractéristique omniprésente de cette société, est fondée sur cette possibilité de multiplier les objets de désir. En sexualité, un événement bien particulier se révélera déterminant pour libérer le désir: l'apparition de Brigitte Bardot. Ses films de la fin des années 50 et du début des années 60 dédramatisent la sexualité, la sortent du démoniaque pour la rendre au naturel, au ludique, à la joie de vivre, à l'innocence : "Avec son jeu d'actrice, la sexualité féminine atteint un degré de franchise et de naturel qui corrige véritablement, peut-être pour la première fois dans le cinéma érotique, l'image de la femme forgée dans |"Occident chrétien' ... Cette féminité indivisible, qu'on ne peut arbitrairement ni simplifier, ni non plus aisément démoniser ou caricaturer, était certainement un message" [10]. Après Brigitte Bardot, la véhémence des moralistes contre la sexualité n'était plus crédible ...

\section{LE DÉSIR AUJOURD'HUI}

Le progrès des connaissances dans de multiples domaines nous a amenés aujourd'hui à concevoir le désir d'une autre façon.

La physiologie a beaucoup progressé, et elle explique désormais que le cerveau humain est concerné par le désir à ses trois niveaux d'intégration : le cerveau primitif reptilien, avec l'hypothalamus, "commande toujours chez l'homme les instincts fondamentaux de fuite, d'agressivité, de désir sexuel, de colère, de défense de notre territoire". Le système limbique se développe avec les mammifères, et intègre les émotions élaborées nécessaires à leur vie sociale plus complexe ; il colore d'émotions les expériences, permettant l'apprentissage. Le troisième et dernier venu, le néocortex, régule l'humeur et contrôle les pulsions, les soumettant à la raison et aux règles de la vie sociale, à ses critères de valeur et de jugement [25]. Le désir est donc profondément enraciné en notre être, avec une composante de réflexe de survie et de réaction automatique émotionnelle sur laquelle vient plus ou moins bien se greffer la composante logique, réfléchie, tenant compte du contexte situationnel. 
Cette explication du fonctionnement cérébral, physiologique et non morale, bouleverse la psychologie et la philosophie et les conduit à donner une autre place au désir. Loin d'être cette source de désordre systématique à fuir et à mépriser, ou bien ce signe d'animalité à réprimer énergiquement, le désir est accepté comme la source des activités humaines, le stimulant de cette curiosité sans limite, caractéristique de l'espèce, qui l'a fait évoluer bien plus que toutes les autres, lui a permis d'acquérir des compétences bien plus variées.

Sur le plan individuel, le désir sexuel nourrit l'enrichissement de la vie sexuelle. Comme pour toutes les autres activités humaines, le désir est soumis aux règles de notre vie sociale, qui ne sont que deux : la liberté de chacun s'arrête où commence celle d'autrui, et la sexualité est libre entre adultes consentants. Tout le reste est affaire de goût et ne reçoit qu'abusivement une connotation morale. Le constat s'impose : alors que les animaux se contentent de répéter des comportements instinctifs pour se nourrir, se protéger et se reproduire, les humains ont de tout temps développé un jeu avec ces besoins fondamentaux. Ainsi le travail effectué pour enrichir les sensations et les plaisirs issus de l'obligation biologique de se nourrir est immense et a abouti au raffinement du désir de manger et de boire ; l'obligation de se protéger est à la source d'un jeu sur les demeures et les vêtements, entretenant des désirs sensuels sans commune mesure avec ce qui se vit dans le monde animal. De même, la nécessité biologique de la reproduction a suscité une exploration des comportements qui prennent en compte la capacité du corps à éprouver et à donner des sensations agréables. Le désir sexuel serait ainsi stimulé par la recherche des plaisirs procurés par le corps : passer du temps à jouer avec ce corps est donc une occupation spécifiquement et profondément humaine, comme passer du temps à préparer la nourriture et à la consommer, comme passer du temps à écouter de la musique, à contempler un paysage ou des tableaux, à humer les odeurs de la nature ou à se parfumer. Toutes ces activités ont été inventées au cours des temps par un mammifère particulièrement curieux d'explorer tout ce qu'il pouvait découvrir sur lui et sur le monde. Nourrir et cultiver son désir sexuel est aussi légitime que cultiver ses dons musicaux, ses capacités sportives, son talent culinaire, sa créativité artistique ... De même qu'il n'y aurait rien d'admirable pour un être humain à retourner vivre nu dans un terrier, et à se nourrir de fruits cueillis ou de racines déterrées, de même ce serait renoncer à son humanité que de se contenter de s'accoupler en récusant toutes les possibilités offertes par l'évolution du désir sexuel humain [12 : 88-126].

En particulier aujourd'hui, dans ce domaine du désir, les couples contemporains ont à relever un défi inédit à cette échelle : celui de la durée de la vie sexuelle de couplesamants. La routine ou la monotonie qui s'installent dans la sexualité d'un couple qui dure ont toujours été contrebalancées par la présence de maîtresses et d'amants, ou par le recours à la prostitution. Or la fin du XXè siècle voit à la fois s'allonger la durée de la vie commune et disparaître, en France, le recours socialement reconnu à la prostitution. Pour vivre la variété des jeux sexuels, pour explorer les émotions que la sexualité peut permettre, pour tester ses limites dans ce domaine, les Français recouraient autrefois aux pensionnaires des maisons spécialisées ("il y a des femmes pour ça !"). Aujourd'hui, l'homme demande à sa compagne, la femme demande à son compagnon, changement de position et de lieu, fellation, cunnilinctus, rapport anal, usage d'objet, et cela de façon de plus en plus naturelle comme le montrent les chiffres de l'évolution de ces pratiques. Certains veulent aller plus loin, avec les jeux dits sado-masochistes (alors qu'ils font simplement partie de toutes les activités humaines ludiques où l'effort, la souffrance que l'on s'impose, la recherche d'émotions fortes, sont des étapes nécessaires pour la qualité du plaisir final), ou avec "l'échangisme". Vaut-il mieux aller voir les prostituées pour se faire fouetter ou pour faire l'amour à trois ou à quatre, ou plutôt tenter de vivre ces émotions en couple avec la personne que l'on aime, avec qui l'on veut tout partager dans sa vie ? Les risques sont réels, pas plus toutefois que ceux qu'affrontaient les couples des périodes précédentes, mais le projet est intéressant : ne pas dissocier les êtres selon leurs diverses fonctions (celui ou celle qui nous donne le plaisir différant de qui nous donne l'amitié, ou l'amour, ou l'enfant, ou la position sociale ...), mais trouver la personne avec qui vivre le plus de liens possible. Et si nos contemporains tâtonnent dans leur recherche sexuelle actuelle, c'est parce qu'aucune route n'est déjà tracée, qu'il y a tout à inventer. Ce n'est pas parce qu'ils auraient mieux fait de ne pas bouger ...

\section{CONCLUSION}

J'ai pris plaisir à vous entraîner dans cette promenade à travers les siècles parce que j'aime cette fraternité du désir qui nous fait humains depuis la nuit des temps et nous rend nos ancêtres si proches, alors que tant d'éléments de nos vies sont complètement différents. Mais il s'avère que le désir a toujours suscité des attitudes contradictoires, entre fascination et répulsion. Même aujourd'hui oủ l'on démonte son mécanisme physiologique d'action, et où l'on constate l'importance de sa présence, le désir reste dénigré, voire nié, par certains de nos contemporains.

Si nous croyons que c'est le désir qui nous distingue des animaux, et nous fait humains, alors il ne faut pas prendre à la légère les comportements et les discours actuels dirigés contre lui (que ce soit la défense d'un voile censé mettre à l'abri du désir, ou celle d'une sexualité qui se confondrait avec l'amitié).

Et chacun d'entre nous a, pour être digne de ces ancêtres qui ont su détacher le désir des périodes de rut ou de chaleurs et nous ont légué ce précieux héritage de liberté, le devoir de parfaire l'humanité en lui, en enrichissant son désir. 


\section{REFERENCES}

1. ALBERT LE GRAND : De Animalibus. Editions Stadler, 1916$1920:$ IX, 1, 1, 7.

2. ARISTOPHANE : Lysistrata. Folio-Gallimard, 1966.

3. AUGUSTIN St : Le bonheur conjugal. Rivages Poche, 2001:75.

4. BASILE St : Sur la renonciation du monde. Patrologie Grecque : 30 : 669-810.

5. BOTTÉRO J. : L'Amour libre à Babylone. L'Histoire, Hors Série $n^{\circ} 5$, juin $1999: 8-13$.

6. BRANTOOME : Les Dames galantes. Le Livre de Poche, 1990.

7. CASANOVA : Histoire de ma vie. Robert Laffont, 1993.

8. CATÉCHISME DE L'ÉGLISE CATHOLIQUE. Mame-PIon, 1992.

9. DESPARS J. : Commentaire au Canon d'Avicenne. Lyon, 1948.

10. DREWERMANN E. : Fonctionnaires de Dieu. Albin Michel, $1993: 446$.

11. FERROUL Y., DRIZENKO A., BOURY D. : Médecin et médecine. Champion, $1997:$ 39-56.

12. FERROUL Y. : Médecins et sexualités. Ellipses, 2002.

13. HÉLOÏSE et ABÉLARD : Lettres et vies. Garnier-Flammarion, 1996.

14. HÉRONDAS : Les Amies ou les intimes. In : Mimes. Les Belles Lettres, 1928 (2003).

15. JUVÉNAL : Satires. Les Belles Lettres, 1931 : VI : 398-412.

16. LACLOS CHODERLOS DE : Les Liaisons dangereuses. Garnier-Flammarion, 1965.

17. MARTIAL : Épigrammes. Les Belles Lettres, 1930, VI : 67.

18. MENDEL G. : La Psychanalyse revisitée. La Découverte, $1988(1998): 21$

19. OVIDE : L'Art d'aimer. Les Belles Lettres, 1960 (Le Livre de Poche, 1963).

20. PONTAS : Dictionnaire de cas de conscience. Paris, 1847.

21. RANKE U. : Des Eunuques pour le royaume des cieux. Laffont, 1990.

22. RIBBING S. Dr : L'Hygiène sexuelle. Paris, $1911: 83$.

23. SAINT-SIMON : Mémoires. Gallimard, $1983 ; 2,1311$.

24. SAVONAROLE M. : Practica Major. Venise, 1547.

25. SCHWOB M. : De l'Amour plein la tête, ou la biologie de l'amour. Hachette, 1984.

26. SÉNĖQUE : Du Mariage.

27. SUÉTONE : Vie des douze Césars. Les Belles Lettres, 1931 (2002).

28. ZELDIN TH. : Histoire des passions françaises, I. Ambition et amour. Seuil, 1978 : 333-365.

Communication au $X X I^{\circ}$ Congrès de la Société d'Andrologie de Langue Française, Clermont-Ferrand, 9-11 Décembre 2004.

Manuscrit reçu : janvier 2005 ; accepté janvier 2005

\section{ABSTRACT}

\author{
A history of desire
}

Yves FERROUL

The striking feature of the history of sexual desire is the contrast between the omnipresence of sexual desire in human societies, regardless of the period considered, and the negative representation constantly and systematically given to this desire by philosophers and religious leaders.

Sexual desire was automatically rejected by those who were disgusted by the animal side of man. The Stoics of the first century A.D., rapidly followed by theologians of the young Christian religion, were convinced that animals only act to satisfy their physical needs, but that moral man had to distinguish himself from the animals by refusing to act simply for pleasure. For Cicero and Seneca "seeking an act because of the pleasure it procures is an abuse". They applied this principle to food as well as bodily care: unthinking animals live to eat, while moral humans eat to live; food and maintenance of the body ensure health but do not provide pleasure. Similarly, sexuality is only justified for reproductive purposes.

However, as already observed by Saint Augustine, this is not the way in which human beings act, and this Church Father had to admit that he had never met a husband able to claim that he "only had sexual intercourse in the hope of fathering".

Other thinkers were more moderate and distinguished acceptable desire, kept under the control of reason and leading to positive action, from degrading, irrational desire ending in passion (always considered to be unreasonable). Only the second form of desire must be avoided; the first leads to happiness and fulfilment and remains the force that usually drives human action.

Christian thinkers of the Middle Ages, attentive to the real life of believers, finally accepted that the human characteristic of memory is one of the most beautiful gifts of God, allowing man to remember past pleasures and therefore to rightfully experience the desire to repeat these pleasures: coitus without a reproductive purpose therefore appears to be part of the divine plan. A theologian, in contrast with dominant religious opinion, even declared "if someone eats a pear or a beautiful fruit only for the pleasure, it is not a sin. The same applies when a man has intercourse with his wife only for the pleasure".

However, far from theoretical discussions, individuals declared their deep attachment to desire, during the Renaissance, at the court of the Sun King, in salons of the Enlightenment or even, in contrast with a widely held belief, in the new industrial world of the 19th century.

Today, while religious authorities still generally have a Stoic view of pleasure and desire, modern thought has advanced in its understanding of the place of desire in 
human experience and recognizes it an essential driving role responsible for fulfilment and a powerful stimulus for all forms of creativity.

The history of sexual desire therefore corresponds to the steps of enrichment of basic animal instinct by the human species and the individual and social forms of expression of this desire.

Key words : sexual desire, human sexuality, pleasure 